BULL. AUSTRAL. MATH. SOC.

VOL. $28(1983), 331-337$.

\title{
EXISTENCE OF LIMIT CYCLES \\ FOR A CLASS OF AUTONOMOUS SYSTEMS
}

\author{
ANTHONY SOFO
}

A proof is given for the existence of at least one stable periodic limit cycle solution for the polynomial non-linear differential equation of the form

$$
\ddot{x}+\rho(\dot{x}, x)+\eta(x)=0,
$$

in some cases where the Levinson-Smith criteria are not directly applicable.

\section{Introduction}

The importance of studying periodic solutions, of differential equations, is best illustrated by the remark of Poincaré [6]:

"D'ailleurs, ce qui nous rend ces solutions périodiques si précieuses, c'est qu'elles sont, pour ainsi dire, la seule brećhe par oú nous puissions essayer de pénétrer dans une place jusqu'ici réputée inabordable."

The aim of this exposition is to prove the following theorem.

THEOREM 1. The phase-plane system

(2)

$$
\left\{\begin{array}{l}
\dot{x}=\alpha x+y, \\
\dot{y}=-g(x)-h(y),
\end{array}\right.
$$

Received 11 July 1983. The author would like to acknowledge the helpful discussions held with Dr A. Jones.

Copyright Clearance Centre, Inc. Serial-fee code: 0004-9727/83 $\$$ A2.00 +0.00 
where

$$
g(x)=\sum_{i=0}^{2 n} \beta_{i} x^{i+1}
$$

and

$$
h(y)=\sum_{i=0}^{2 n} \gamma_{i} y^{i+1}
$$

has at least one stable limit cycle solution for $n \in J^{+} \mid\{0\}$ if

$$
\begin{aligned}
& \text { (i) }\left\{\frac{g(x)+h(-\alpha x)}{x}\right\}>0 \text {, for alz } x \in I R \mid\{0\}, \\
& \text { (ii) } \beta_{0}>0, \gamma_{0}<0, \\
& \text { (iii) } 0 \leq \alpha<1, \\
& \text { (iv) } 0<\gamma_{2 n}<(1-\alpha) \beta_{2 n} .
\end{aligned}
$$

The system (2) may be expressed as a second order differential equation of the form ( 1 ), where

$$
\rho(\dot{x}, x)=h(\dot{x}-\alpha x)-\alpha \dot{x}, n(x)=g(x) .
$$

A set of conditions for the existence of at least one stable periodic solution to the system

$$
\left\{\begin{array}{l}
\dot{x}=y, \\
\dot{y}=-n(x)-\rho(y, x) y,
\end{array}\right.
$$

has been given by Levinson and Smith [3], and improved by Dragilev [2] and Villari [9]. Unfortunately some of those conditions for system (5) cannot be directly applied to the system (2). Similarly the conditions for the existence of at least one stable limit cycle for the generalized Lienard equation

$$
\ddot{x}+\dot{x} f(x)+g(x)=0
$$

are not directly applicable to the system (2).

In the case where $\alpha=0$, condition (i) of the theorem will be replaced with the requirement that

$$
\left\{\frac{g(x)}{x}\right\}>0, \text { for all } x \in I R \mid\{0\},
$$


and the second order differential equation becomes

$$
\ddot{x}+h(\dot{x})+g(x)=0 .
$$

Various cases of the above equations have been investigated, using different methods from the ones employed in this exposition, by Reissig [7], Parker van Zandt [5], Sansone and Conti [8] and Zhifen [10], but none of their results cover the cases included in the theorem.

\section{Preliminary results}

DEFINITION 1. Cycle without contact.

Given the system (2),

$$
\left\{\begin{array}{l}
\dot{x}=\alpha x+y, \\
\dot{y}=-g(x)-h(y),
\end{array}\right.
$$

where $g(x)$ and $h(y)$ are given by (3) and (4) respectively, let $C$ be a simple smooth closed curve in an annular region $G \subseteq I R^{2}$ and $M$ any point on $C$. The curve $C$ does not have contact with the trajectories of the system (2) at the point $M$, if $C$ is not tangent to a trajectory of the system (2) at $M$. Hence, a simple smooth closed curve $C$ is called a cycle without contact to the system (2) if

(i) there are no singular points on $C$,

(ii) the curve $C$ does not have contact at any of its points.

DEFINITION 2. A topographic system is defined to be a family of nonintersecting simple smooth closed curves

$$
W(x, y)=C
$$

lying interior to one another and filling out some annular region $G$.

THEOREM 2. Given the system (2),

$$
\begin{aligned}
& \dot{x}=\alpha x+y, \\
& \dot{y}=-g(x)-h(y) .
\end{aligned}
$$

Let $G \subseteq I R^{2}$ be a doubly connected annular region bounded by two cycles without contact $C_{0}$ and $C_{1}, G$ contain no singular points, and $a$ finite number of closed paths and suppose the trajectories of (2) crossing 
$C_{0}$ and $C_{1}$ enter $G$ with increasing time $t$. Then the number of stable limit cycles in $G$ is greater by one than the number of unstable limit cycles. In particular, under the above conditions, the system (2) has at least one stable limit cycle in $G$.

The proof of this theorem for any general analytic two-dimensional dynamic system can be found in [1] or [4].

\section{Proof of Theorem 1}

The singularities of the system (2) occur when $y=-\alpha x$ and

$$
\sum_{i=0}^{2 n}\left\{\beta_{i}+\gamma_{i}(-\alpha)^{i+1}\right\} x^{i+1}=0
$$

therefore condition ( $i$ ) is sufficient to ensure a unique singularity at the origin $(0,0)$ of the phase plane, the linearized behaviour of the trajectories of system (2) in the neighbourhood of the origin is classed as

(a) an unstable node, for $\beta_{0} \geq\left(\frac{\alpha+\gamma_{0}}{2}\right)^{2}$, or

(b) an unstable focus (source), for $\beta_{0}<\left(\frac{\alpha+\gamma_{0}}{2}\right)^{2}$ since $\alpha \geq 0, \beta_{0}>0$ and $\gamma_{0}<0$.

The Poincaré index of the singularity at the origin is +1 . Consider the topographic family of cycles without contact,

$$
W(x, y)=\frac{y^{2}}{2}+G(x)=C_{0}
$$

where

$$
G(x)=\int_{0}^{x} g(\xi) d \xi
$$

the time derivative of (6) along trajectories of the system (2) is

$$
\dot{W}(x, y)=\alpha x g(x)-y h(y) \text {. }
$$

Since $g(x)$ and $h(y)$ are continuous and $\alpha \geq 0, \beta_{0}>0$ and $\gamma_{0}<0$, then there exists a neighbourhood of $(0,0)$ in which 


$$
\dot{W}(x, y)>0
$$

Hence for arbitrary small curves, near the origin it follows that $\dot{W}(x, y)>0$ on the closed curves of the family $W(x, y)=c_{0}$, for $c_{0}$ arbitrarily small.

Now consider

$$
V(x, y)=\frac{y^{2}}{2}+x y+G(x)=C_{1}
$$

$G(x)+\infty$ for $x \rightarrow \pm \infty$ and therefore (8) will be a family of closed curves for a large enough $C_{1}$; that is,

$$
y(x)=-x \pm \sqrt{2 C_{1}-\left(2 G(x)-x^{2}\right)} .
$$

Now $\dot{V}(x, y)=y(\alpha x+y)-x(1-\alpha) g(x)-(y+x) h(y)$. In polar co-ordinates,

(9) $\dot{V}=r^{2} \sin \theta(\alpha \cos \theta+\sin \theta)$

$$
-\sum_{i=0}^{2 n} r^{i+2}\left\{(1-\alpha) \beta_{i} \cos ^{i+2} \theta+\gamma_{i} \sin ^{i+1} \theta \cos \theta+\gamma_{i} \sin ^{i+2} \theta\right\} \text {, }
$$

$r>0$ and $\theta \in[0,2 \pi]$.

Let

$$
H_{2 n}(\theta)=r^{2 n+2}\left\{(1-\alpha) \beta_{2 n} \cos ^{2 n+2} \theta+\gamma_{2 n} \sin ^{2 n+1} \theta \cos \theta+\gamma_{2 n} \sin ^{2 n+2} \theta\right\}
$$

and investigate the sign of $H_{2 n}(\theta)$, since this leading term, for $r$ sufficiently large, will determine the sign of $\dot{V}(x, y)$.

Let $m=\sin \theta$; hence $|m| \leq 1$. Therefore

$$
H_{2 n}(\theta)=(1-\alpha) \beta_{2 n}\left(1-m^{2}\right)^{n+1}+\gamma_{2 n} m^{2 n+2} \pm \gamma_{2 n} m^{2 n+1}\left(1-m^{2}\right)^{\frac{1}{2}}
$$

For $0 \leq \alpha<1$, in (10) it will be sufficient to show that

$$
(1-\alpha) B_{2 n}\left(1-m^{2}\right)^{n+1}+\gamma_{2 n} m^{2 n+2}>\gamma_{2 n} m^{2 n+1}\left(1-m^{2}\right)^{\frac{1}{2}} \text {. }
$$

For $m^{2} \geq \frac{1}{2}$, then $m \geq\left(1-m^{2}\right)^{\frac{1}{2}}$ and hence

$$
\gamma_{2 n} m^{2 n+2} \geq \gamma_{2 n} m^{2 n+1}\left(1-m^{2}\right)^{\frac{3}{2}}
$$


Inequality (1l) now follows since the other term on its left hand side is positive.

For $0 \leq m^{2}<\frac{1}{2}$ then $\left(m^{2}\right)^{n+\frac{1}{2}}<\left(1-m^{2}\right)^{n+\frac{1}{2}}$ and hence by condition (iv) of the theorem

$$
(1-\alpha) B_{2 n}\left(1-m^{2}\right)^{n+1}>\gamma_{2 n} m^{2 n+1}\left(1-m^{2}\right)^{\frac{1}{2}} .
$$

Again inequality (11) follows since the other term on its left hand side is positive. Therefore from $(10), H_{2 n}(\theta)>0$ for all $\theta \in[0,2 \pi]$ and from (9), $\dot{V}(x, y)<0$ for a family of closed curves $V(x, y)=C_{1}$ for sufficiently large $C_{1}$. By invoking Theorem 2, we see that the system (2) has at least one stable limit cycle solution.

REMARK. Condition ( $i$ ) of the theorem still remains valid if

$$
B_{i}=\gamma_{i}=0, i=1,2, \ldots, 2 n-1,
$$

since conditions $(i i)$ and $(i v)$ determine the other constants. Hence $g(x)$ can be taken as an add function in $x$ and $h(y)$ can be taken as an odd function in $y$.

\section{References}

[1] A.A. Andronov, E.A. Leontovich, I.I. Gordon and A.G. Maier, Qualitative theory of second-order dynamic systems (John Wiley \& Sons, New York, London, 1973).

[2] A.D. Dragilev, "Periodic solutions of the differential equation of non-linear oscillations", Prikl. Mat. Meh. 12 (1952), 85-88.

[3] N. Levinson and O.K. Smith, "A general equation for relaxation oscillations", Duke Math. J. 9 (1942), 382-403.

[4] N. Minorsky, Nonlinear oscizlations (Van Nostrand, Princeton, 1962).

[5] J. Parker Van Zandt, "Oscillating systems by resistance proportional to the square of the velocity", Phys. Rev. 15 (1919), 415-443.

[6] H. Poincaré, Oeuvres (Gauthier-Villars, Paris, 1928). 
[7] R. Reissig, "Selbsterregung eines einfachen Schwingers", Math. Nachr. 15 (1956), 191-196.

[8] G. Sansoni and R. Conti, Non-linear differential equations (Macmillan, New York, 1964).

[9] G. Villari, "On the existence of periodic solutions for Lienard's equation", Nonlin. Anal. 7 (1983), 71-78.

[10] Z. Zhifen, "Theorem of existence of exact $n$ limit cycles in $|\dot{x}| \leq(n+1) \pi$ for the differential equation $\ddot{x}+\mu \sin \dot{x}+x=0$ ", Sci. Sinica 23 (1980), 1502-1510.

Department of Applied Mathematics,

La Trobe University,

Bundoora,

Victoria 3083,

Australia. 